\title{
Naloxone Training and Distribution Program in an Urban Collegiate Setting scovonice
}

\author{
Andrew Lam, EMT; Samantha Steeman, EMT; Gabrielle Ramirez, EMT; Joshua Glick, MD
}

POSTER PRESENTATION ABSTRACT | PROGRAM DEVELOPMENT \& EVALUATION CATEGORY

Introduction: The opioid crisis plaguing America has been called Philadelphia's "greatest public health crisis in a century." Numerous members of the Penn and West Philadelphia communities live and work in areas where opioid overdoses will continue to occur. These bystanders have personally witnessed overdoses, but lacked either the training or means to effectively respond in multiple cases that Penn's student-run Medical Emergency Response Team (MERT) has been made aware of. Naloxone (Narcan) can rapidly reverse the effects of opioid overdoses. However, one pack can cost upwards of $\$ 130$, and free, in-person naloxone administration trainings by qualified instructors are rare.

Program Development \& Implementation: MERT worked with physicians from Penn Medicine's Emergency Department to develop a 1-1.5 hour bystander naloxone training curriculum. The program consists of opioid crisis information, how to detect an overdose and administer naloxone, and integrates rescue breathing and CPR. MERT forged a partnership with Philadelphia's Department of Public Health to acquire a renewable Narcan supply for distribution to trainees who face financial, insurance-based, or societal barriers in obtaining naloxone. With an initial 96-dose Narcan supply, MERT provided pilot trainings to Penn Social Policy and Practice, Medicine, Nursing, and Education graduate students.

Program Evaluation: In anonymous post-training surveys since March 2019, the trainings' effectiveness received an average score of 4.83 ( $\mathrm{SD}=0.41$ ) on a Likert scale, with "1" representing "poor" and "5" representing "excellent." Participants who use Narcan supplied by MERT can report their use and receive another dose, allowing for continued evaluation.

Discussion/Conclusions: To our knowledge, this is the only collegiate EMS-run bystander naloxone training program to date. Collegiate agencies can play crucial roles in overdose awareness and prevention. Due to initial success, MERT will collaborate with Penn's Division of Public Safety to teach integrated CPR/AED/Narcan trainings and continue the existing program, while expanding into the greater West Philadelphia community.

Author Affiliations: From Medical Emergency Response Team, University of Pennsylvania - in Philadelphia, PA, USA (A.L., S.S. \& G.R.). From Department of Emergency Medicine, Hospital of the University of Pennsylvania, University of Pennsylvania - in Philadelphia, PA, USA (J.G.).

Address for Correspondence: Samantha Steeman | Email: outreach@pennmert. org

Conflicts of Interest/Funding Sources: By the JCEMS Submission Declaration Form, all authors are required to disclose all potential conflicts of interest and funding sources. The authors declared that they have no conflicts of interest. The authors declared that they did not receive funding to conduct the program or research associated with this work.

Ethical Compliance: The authors attest that the research associated with this abstract was conducted in accordance with the JCEMS Ethics Guidelines.

Submission History: Received January 13, 2020; accepted for presentation and publication February 26, 2020.

Poster Presentation: This abstract was presented as a poster at the Academic Poster Session of the 27th Annual Conference of the National Collegiate Emergency
Medical Services Foundation; February 29, 2020; Boston, MA, USA. The authors received the Second Place Award in the Poster Presentation Competition.

Published Online: December 31, 2020

Published in Print: December 31, 2020 (Volume 3: Issue 2)

Reviewer Information: In accordance with JCEMS editorial policy, poster presentation abstracts undergo double-blind peer-review by at least two reviewers (JCEMS Editorial Board members and/or independent reviewers) prior to acceptance for presentation and publication. JCEMS thanks the anonymous reviewers who contributed to the review of this work.

Copyright: () 2020 Lam, Steeman, Ramirez \& Glick. This is an open access article distributed under the terms of the Creative Commons Attribution 4.0 International (CC BY 4.0) License, which permits unrestricted use, distribution, and reproduction in any medium, provided the original author and source are credited. The full license is available at: https://creativecommons.org/licenses/ by $/ 4.0 /$

Electronic Link: https://doi.org/10.30542/JCEMS.2020.03.S1.02 